\title{
SHAPE FROM SHADING USING DISCRETE POLYNOMIALS
}

\author{
P.K. Bora, Y.V. Venkatesh and K.R. Ramakrishnan, \\ Department of Electrical Engineering, \\ Indian Institute of Science, \\ Bangalore 560012
}

ABSTRACT

Given a monocular image, we propose a new method for extracting the shape of objects from the pixel intensities. The problem of solving the partial differential equation corresponding to the reflectance map is reduced to that of solving a finite set of nonlinear algebraic equations. The method involves patchwise approximation of the surfaces in the scene by discrete polynomials. Distinctive features of the method are as follows: a) shape extraction without initial depth information of any form, (b) Handling of discontinuity lobtained by a zero-crossing operator) by splitting the region into multiple patches around the discontinuities contour and fitting the depth polynomial separately in each patch, (c) Algebraic evaluation of the quadratic polynomial coefficients in the case of identical source and viewer direction, and (d) Facility to integrate stereo information.

\section{Introduction}

The problem considered in this paper is one of recovering surface information from a monocular image of the scene, more specifically from the shading caused by the difference of orientation in different parts of the object. This is known as the "Shape from Shading (SFS)" problem. The mathematical formulation of the problem in terms of the relationship between image brightness and the surface orientation is due to Horn [1]. In Fig.1 which depicts the imaging geometry for the SFS problem, a Lambertian surface is assumed to be illuminated from a distant point source located at a known direction $\left(p_{s}, q_{s},-1\right)$. With furhter assumption. of orthographic projection, the image formation is governed by

$$
E(x, y)=\frac{p\left(1+p p_{s}+q q_{s}\right)}{\sqrt{\left(\left(1+p^{2}+q^{2}\right)\left(1+p_{s}{ }^{2}+q_{s}{ }^{2}\right)\right)}}
$$

wherepis the albedo constant (assumed to be 1) and $(p, q,-1)$ the surface normal vector. The surface normal parameters $p$ and $q$ are the first order partial derivatives of depth $z(x, y)$ with respect to $x$ and $y$ respectively. In a more general case the observed, intensity is related to a reflectance map $R(p, q)$ which is obtained from the particular scene illumination, surface reflectance property and imaging geometry [2], [3].

$$
E(x, y)=R(p, q)
$$

As evident from (1.1) and (1.2), the shape from shading amounts to solving a first order nonlinear partial differential equation.

Horn's [1] characteristic strip method tries to solve the image irradiance equation by replacing it by an equivalent set of five ordinary differential equations. These 'Chirpit's equations' [4] express each of the variables $x, y, z, p$ and $q$ in terms of the derivative with respect to a dummy parameter s. Starting from a point of known orientation $\left(p_{0}, q_{\emptyset}\right)$, the parameter $s$ is varied progressively to obtain new points and their corresponding orientation and depth. The Characteristic strip method suffers from the drawback of error accumulation owing to the presence of noise [5].

Ikeuchi and Horn [5] incorporates the occluding boundary information and surface smoothness constraint and reformulates the problem in the light of Variational Calculus. The inability of the gradient space to handle the occluding contour (either $p$ or $q$ or both become infinite) led to the use of stereographic projection coordinates $(f, g)$. The problem so posed is to find a smooth surface that minimizes the square sum error between the observed image and the reflectance map. Mathematically, the problem is to minimize

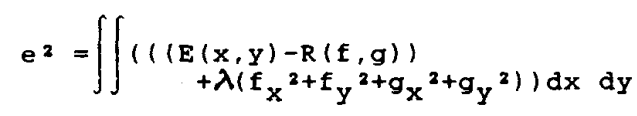

where the subscripts $x$ and $y$ denote the first order partial derivatives and $\lambda$ is the parameter to counter the deviation from smoothness. The Euler equations corresponding to the above problem result in

$$
\begin{aligned}
& \nabla^{2} f=(E(x, y)-R(f, g)) \frac{\partial R}{\partial f} \\
& \nabla^{2} g=\left(E(x, y)-R(f, g), \frac{\partial R}{\partial g}\right.
\end{aligned}
$$

These Poisson type equations are numerically solved with the known values of $f$ and $g$ at the occluding boundary. Several authors have improved upon the Ikeuchi-Horn algorithm by way of incorporating integrabillity constraint [6], and including other multiple cues like binocular and photometric stereo, etc. $[7],[8]$. 
Another approach proposed by Pentland (9) assumes the surface to be locally umbilical which results in interpretation of the surface from the image and its first and second derivatives. The spherical assumption is an inadequate model for any general surface. Pentland [10], [11] proposes a linear reflectance function on the basis of Taylor series approximation of $R(p, q)$ about the point $(p=0, q=0)$. He uses a Fourier transform method to recover the depth information. from the image spectrum. However, to apply his method the surface orientation should be restricted by $|p|,|q| \ll<$ or the source direction is within $\pm 30^{\circ}$ of the viewer direction.

Coupled inherently with the problem of shape from shading is the problem of "source from shading", i.e., finding the illumination direction from the shaded image. Brooks and Horn [12] give an iterative algorithm to alternately estimate the surface normal and the source direction in a variational formulation of the problem in terms of the surface normal and its first order partial derivatives. The square sum error is minimized with respect to the source vector assuming that the surface normal is known. Simple Lagrange multiplier technique has been applied to get a recursive relation for the source vector in terms of the currently estimated surface normal.

Pentland [9], [13] and Lee and Rosenfeld [14] apply a statistical formulation to the source estimation problem. With the assumption that change in surface normal is isotropically distributed, a least square estimation procedure is proposed with the directional derivatives of the image as given data. In the Lee - Rosenfeld procedure, a joint probability distribution of surface slant and tilt is assumed as originally put forward by witkin [15] in connection with shape estimation from texture.

\section{Shape Recovery by Polynomial approximation}

We assume that the original scene contains objects which could be polyhedral or any other shape. The surface visible to the viewer is approximated by orthogonal polynomials in small patches, the size of the patch being dependent on the prior information about the scene obtained by preprocessing the image: whether the scene contains smooth or discontinuous surfaces and whether the objects are occluded.

Consider a patch $R$ of size $(2 \mathrm{M}+1) \times(2 \mathrm{M}+1)$ in an image of size $N \times N$ with the centre of the patch having co-ordinates $(0,0)$. We assume that original object(s) in the scene have a depth function $z(x, y)$ given by

$$
\begin{aligned}
z(x, y)= & \sum_{i, j} a_{i j} \cdot P_{i j}(x, y) \\
& \text { for all } x, y \in R
\end{aligned}
$$

where $P_{j j}(x, y)$ belongs to a basis set of discrete polynomials.[21]
From $(2.1)$, we have

$$
\begin{aligned}
& p=\frac{\partial z}{\partial x}=\Sigma a_{i j} \cdot \frac{\partial P_{i j}}{\partial x} \\
& q=\frac{\partial z}{\partial y}=\Sigma a_{i j} \cdot \frac{\partial P_{i j}}{\partial y}
\end{aligned}
$$

The image irradiance equation (1.1) now becomes

$$
\begin{aligned}
& E(x, y)= \\
& \frac{1+p_{s} \Sigma a_{i j} \cdot \partial p_{i j} / \partial x+q_{s} \Sigma a_{i j} \cdot \partial p_{i j} / \partial y}{\checkmark\left(1+\left(\Sigma \partial p_{i j} / \partial x\right)^{2}+\left(\Sigma \partial P_{i j} / \partial y\right)^{2}\right)\left(1+p_{s}{ }^{2+q_{s}}{ }^{2}\right)} \\
& =R(\underline{a}) \text {, where } \underline{a}=\left|a_{i j}\right|
\end{aligned}
$$

Thus the problem now is to estimate the parameters of the polynomial from the relation $(2.2)$

In a similar model, Pong et. al. [16] proposes a least square formulation of the problem, as

$$
\underset{\underline{a}}{\operatorname{minimize}} e^{2}=\underset{x, y}{\Sigma}|E(x, y)-R(\underline{a})|^{2}
$$

where they consider the polynomial fit in a global region. This global optimization scheme requires a sparse depth information obtained by matching a stereo pair of images. The shading information is used just to assist in the interpolation. This is against the independent nature of the shape from shading problem.

From the Taylor series expansion of depth $z(x, y)$ about a point $\left(x_{0}, y_{0}\right)$ it is obvious that a finite order polynomial approximation is not justified in global sense. A patchwise fit is consistent with theoretical justification. Instead of an ordinary polynomial, an orthogonal polynomial of the same order gives a better fit because of the Bessel's inequality in the Hilbert space. [17].

The advantage of the orthogonal polynomial over the ordinary polynomial is that we can select the order of the polynomial from the analysis of the coefficients. Also, the discontinuity point can be detected from the change in polynomial parameters between two adjacent patches.

\subsection{Shape Estimation}

Case I: Identical source and viewer direction:

Let us first consider the simple case where the light source and the viewer directions coincide. We have

$$
E(x, y)=\frac{1}{\sqrt{\left(1+p^{2}+q^{2}\right)}}
$$


from which we get

$$
p^{2}+q^{2}=\frac{1}{E^{2}(x, y)}-1=f(x, y)
$$

Eq.2.4 is the so called Eikonal equation [18]. Assume that the depth function can be represented by a quadratic polynomial, given by

$$
z(x, y)=a_{00}+a_{10} x+a_{01} y+a_{11} x y+a_{20} x^{2}+a_{02} y^{2}
$$

Note that in $(2.5)$ we have employed an ordinary polynomial instead of an orthogonal one because the partial derivatives are same for both.

Substituting $z$ in $(2.4)$ we get,

$$
\text { where } \begin{aligned}
c_{00}+c_{10} x+c_{01} y+c_{11} x y+c_{20} x^{2}+c_{02} y^{2}=f(x, y) & =c_{00} \\
4 a_{10} a_{20}+2 a_{01} a_{11} & =c_{10} \\
4 a_{01} a_{02}+2 a_{10} a_{11} & =c_{01} \\
a_{11}{ }^{2}+4 a_{20}{ }^{2} & =c_{20} \\
a_{11}+4 a_{02} & =c_{02} \\
4 a_{11}\left(a_{20}+a_{02}\right) & =c_{11}
\end{aligned}
$$

For a patch of size $(2 M+1) \times(2 M+1)$ there are equal number of equations of the form (2.6). Solving the set of equations for the unknown coefficient $c_{i j}$ and using the set of auxiliary equations (2.7) the $a_{j j}$ coefficients can obtained. Substituting the estimated coefficients in $(2.5)$, depth $z(x, y)$ can be estimated.

\section{Case II: General source direction:}

The Eikonal equation formulation is not applicable in case of the general source direction. Auxiliary equations of the type (2.7) can be obtained by means of the various image derivatives at the centre of the patch. The second degree polynomial parameters can then be extracted from the first order and the second order derivatives at the centre of the patch. With $B$ denoting the intensity at the centre of the patch, $\alpha=p_{g} / f\left(1+p_{g^{2}}{ }^{2}+q_{s}{ }^{2}\right), \beta=q_{g} / f\left(1+p_{s}^{2}+q_{s}{ }^{2}\right)$, $\tau=1 / f\left(1+p_{s}{ }^{2}+q_{s}{ }^{2}\right)$ and the subscripts of $x$ and $y$ denoting partial derivatives. These equations are:

$$
\begin{aligned}
& E\left(a_{10}+a_{01}\right) E_{X}+E^{2}\left(2 a_{10} a_{20}+a_{01} a_{11}\right) \\
& =\left(\tau+\alpha a_{10}+\beta a_{01}\right)\left(2 \alpha a_{20}+\beta a_{11}\right) \\
& E\left(a_{10}+a_{01}\right) B_{y}+E^{2}\left(2 a_{01} a_{02}+a_{10} a_{11}\right) \\
& =\left(\tau+\alpha a_{10}+\beta a_{01}\right)\left(2 \beta a_{02}+\alpha a_{11}\right) \\
& \left(B_{X}^{2}+E E_{X X}\right)\left(a_{10}+a_{01}\right) \\
& +4 E E_{X}\left(2 a_{10} a_{20}+a_{01} a_{11}\right) \\
& +E 2\left(2 a_{20}+a_{11}\right)
\end{aligned}
$$

$$
\begin{aligned}
= & \left(2 \alpha a_{20}+\beta a_{11}\right)^{2}\left(B_{y}{ }^{2}+E E_{y Y}\right)\left(a_{10}+a_{01}{ }^{2}\right) \\
& +E E_{y}\left(2 a_{01} a_{02}+a_{10} a_{11}\right) \\
& +E^{2}\left(2 a_{01}+a_{11}\right) \\
= & \left(2 \beta a_{02}+\alpha a_{11}\right)^{2}\left(E_{X} E_{Y}+E E_{X Y}\right)\left(a_{10}{ }^{2}+a_{01}\right)^{2} \\
& +E\left(2 a_{10} a_{20}+a_{01} a_{11}\right) E_{Y} \\
& +2 E^{2} a_{11}\left(a_{20}+a_{02}\right) \\
= & \left(2 \alpha a_{20}+\beta a_{11}\right)\left(2 \beta a_{02}+\alpha a_{11}\right)
\end{aligned}
$$

The image derivatives can be computed from the image data, and the set of equations (2.8) can be solved to extract the polynomial parameters.

3. Numerical solution scheme and experimental Results :

For the case $I$, the $c_{i j}$ coefficients are estimated from a least square fit [19] to $f(x, y)$ data. The system of equations in (2.7) leads to a total of 16 solutions. Out of these only 4 are consistent with image data. These four solutions correspond to one convex, one concave and two saddle-surface solutions. This only confirms the ambiguity of SFS problem. Convexity assumption imposes the constraint $a_{20}<0, a_{2}<0$ and $4 a_{20} a_{02}>a_{11}{ }^{2}$ on the $a$ coefficients and leads to unique solution.

The algebraic solution of the set of auxiliary equations are very difficult for a general light source direction and particularly when the order of the polynomial is large. The various numerical techniques of solutions to nonlinear equations demand an approximate guess of the solution as the initial value. These approximate values are to be obtained either from preprocessing of the image or from the orientation information at the occluding boundary.

At the singular points (points of maximum gray value) and occluding contour. the surface normal can be locally determined. The starting patch can be centred around such a point and computation can be proceeded from this point. However, if the order of the polynomial is not proper, greater error in estimation will be introduced at a point of larger orientation. Our computational experience favours the initial patch to be taken around a singular point. We apply the Newton - Raphson technique [20] for the numerical solution of equations $(2.8)$.

\subsection{Enforcing snoothness and Integration with stereo information}

For a smooth surface, it becomes necessary to preserve the continuity of depth and orientation across patches. This can be achieved by refitting the depth data into a smooth surface.

The depth-map corresponding to stereo information can be used to estimate the $a_{j} j$ parameters of the initial patches takeh 
around a contour where such data are available. These coefficients provide good initial guess for a nearby patch.

\subsection{Computational Results:}

The results of reconstruction, for the following cases, are shown in figures $(2-5)$ :

(i) Synthetic saddle surface; quadratic polynomial fitting over the entire image $\left(p_{s}=0, q_{s}=0\right)$. (Figure 2$)$

(ii) Synthetic partial sphere on a plane; global and patch-wise fit $\left(p_{3}=q_{3}=0\right)$ (Figure 3)

(iii) Natural surface ; quadratic polynomial fitting:

a) A paper cylinder ; (Figure 4)

b) $A$ portion of an orange ; (Figure 5)

\section{Conclusion}

A method has been presented for recovering surface depth from the shading information by patch-wise polynomial fit of the depth. For the simple case of identical source and viewer direction, explicit algebraic technique based on quadratic approximation of the surface is given. For general source direction iterative technique is presented on the basis of orientation information in the singular point in the image. The method is applied to surface recovery from synthetic and real images.

\section{References}

[1]. B.K.P.Horn, Obtaining shape from Shading Information', in The Psychology of Computer Vision, (Ed.), P.H.Winston, Me Graw Hill, NY, 1975.

[2]. B.K.P.Horn, 'Robotic Vision', MIT Press, Cambridge, U.S.A. 1986.

[3] . B.R.P.Horn and R.w.Sjoberg, Calculating the Reflectance Map', Applied Optics. vol. 18, No. 11, pp. 1770-1779, 1979.

[4]. M.G. Smith, Introduction to the Theory of Partial differential Equations', Van Nostrand, 1967.

[5]. R.Ikeuchi and B.K.P.Horn, 'Numerical shape from shading and occluding Boundaries', Artificial Intelligence, Vol. 17, pp. 141-184, 1981.

[6]. R.T.Frankot and R.Chellappa, 'A Method for enforcing Integrabilitity in Shape from Shading Algorithms', IBEB Trans. Pattern Anal. Machine Intell., Vol. PAMI-10, pp.

[7]. H.H.BOlthoff and H.A.Mallot, Integration of depth modules: stereo and shading', Journal of Optical Society of America. vol. 5, No 10, pp 1749-1758 October, 1988
[8]. M.M. Nasrabadi, S.P. Clifford and Yi Liu, - Integration of stereo vision and optical flow by using an energy minimization approach' Journal of Optical society of Mmerica, vol. 6, No 6. Pp 900-907, June,1989.

[9]. A.P.Pentland, 'Local Shading Analysis', IEE: Trans. Pattern Mnal. Machine Inte11., Vol. PAMI-6, pp.170-187, 1984.

[10].A.P. Pentland, 'Shape from Shading: A theory of human perception". Proc. of International Conference of Computer vision, IBEB Society, Dec 5-8, Tarpon Springs, F1.

[11].A.P. Pentland, 'A possible Neural Mechanism for Computing Shape from Shading', Neural Computation, 1, pp. 208-217, 1989 .

[12].M.J.Brooks and B.R.P.Horn, 'Shape and Source from shading', Proc. IJCAI, pp.932-936, 1985.

[13].A.P.Pentland, 'Finding the Illuminant Direction', Journal of Optical Society of America, vol, 72, No 4, pp 448-455, Apri1, 1982

[14].D.Lee and A.Rosenfeld, 'Improved Methods for Estimating shape from shading Using light Source coordinate system. Artificial Intelligence, vol. 26 , pp.125-143, 1985.

[15].A.P.Witkin, 'Recovering Surface Shape and Orientation from Texture, Artificial Intelligence, Vol. 17, No.1-3, pp.17-45, 1981 .

[16].Pong,Haralick and Shapiro, Shape from shading using facet model', Pattern Recognition, vol. 22, No 6, pp 683695,1989 .

[17].R.J. Schilling, H. Lee, 'Engineering Analysis, a vector space approach', Wiley, New York, 1988.

[18].A.R. Bruss, 'The eikonal equation : Some results applicable to computer vision', J1. Mat. Phys. 23, No.5, pp. 890-896,1982.

[19].N. Mohanty.' Random Signals Estimation and Identification', CBS 1987.

[20].Carl E. Pearson, 'Numerical Method in Engineering and science', CBS, 1987.

[21].R.M. Haralick, 'Digital step Edges from Zero Crossing of Second Directional Derivatives', IBBE Trans. Pattern Anal. Machine Intell., Vol. PAMI-6, No.1 pp. $58-68,1984$ 


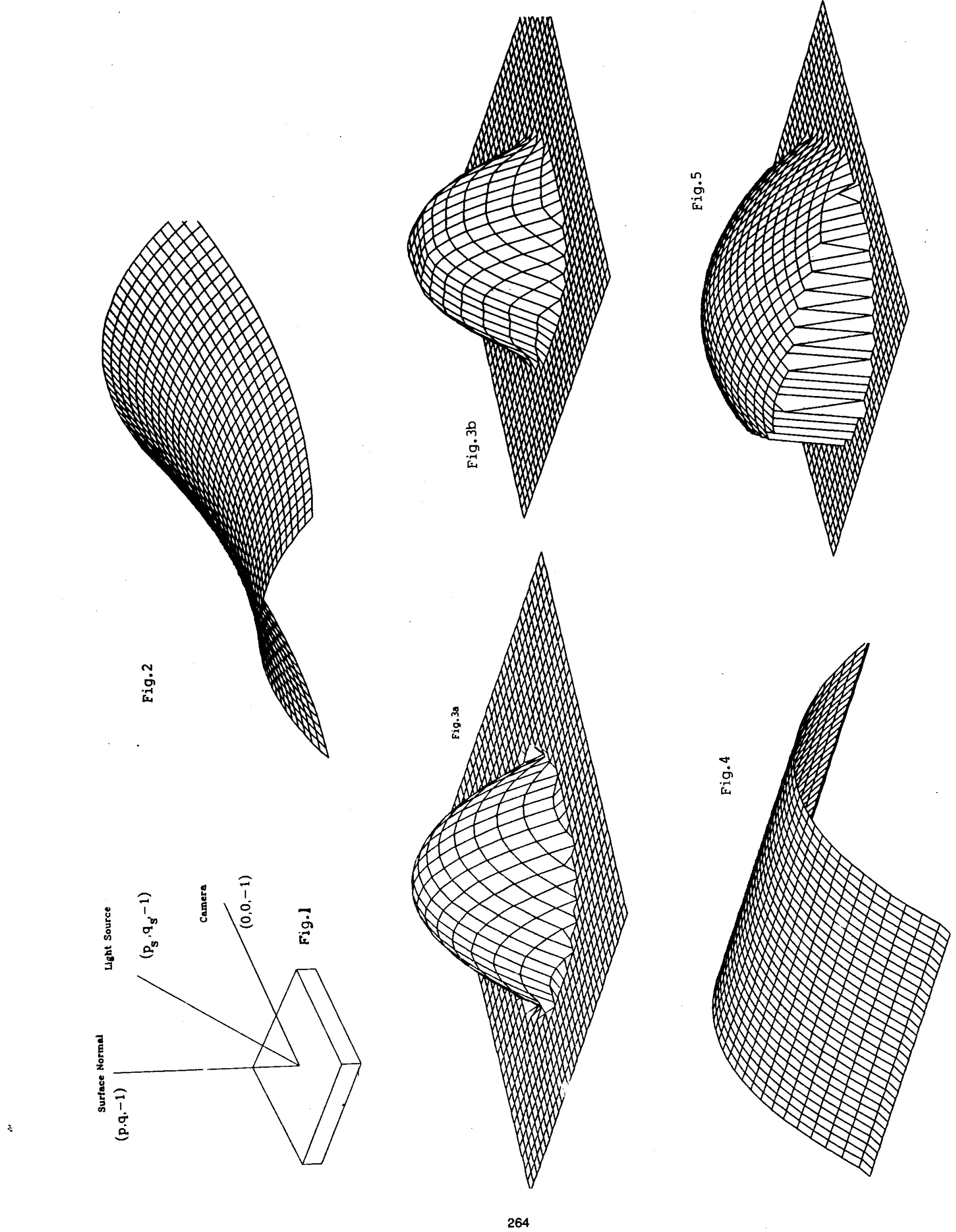

